\title{
Correction to: Safety in Sosa
}

\section{John Greco ${ }^{1}$}

Published online: 27 August 2018

(c) Springer Nature B.V. 2018

\section{Correction to: Synthese https://doi.org/10.1007/s11229-018-1863-z}

Shortly after the publication of this paper, I had the opportunity to discuss related issues with Thomas Grundmann, who convinced me that the final section contains a demonstrable mistake. Specifically, an apt second-order perspective does not convert first-order SSS-relative safety into first-order skill-relative safety, contra to what I claimed. This is because, as Grundmann pointed out, second-order cognitive capacities are themselves to be understood in terms of an SSS structure, and therefore secondorder aptness only guarantees safety from error within such parameters.

What can be saved in the final section is this: Although an apt second-order perspective does not entail first-order skill-relative safety, it moves one toward that result to the extent that the SSS parameters for one's second-order capacities are broader than those for one's first-order capacities. And this is indeed often the case; for example, when second-order reflection on perception is apt within parameters that are broader than those that are appropriate for perception itself. Accordingly, an apt second-order perspective may improve one's epistemic position in this respect, i.e., by moving one closer to skill-relative safety regarding one's first-order beliefs. Moreover, ascent to an apt third-order perspective or beyond yields no analogous benefit, at least not on the first order, and likely not on subsequent orders either, insofar as SSS parameters for higher-order capacities do not likely trend broader.

The original article can be found online at https://doi.org/10.1007/s11229-018-1863-z.

\footnotetext{
$凶$ John Greco

jgreco2@slu.cdu

1 Leonard and Elizabeth Eslick Chair in Philosophy, Department of Philosophy, Saint Louis University, 3800 Lindell Blvd., Adorjan Hall 306, Saint Louis, MO 63108, USA
} 